\title{
SCALABLE BIOMETRIC TRAVEL TOKEN WITHOUT BARRIERS TO ACCESS
}

\author{
NIOSHA KAYHANI \& STEFFEN REYMANN \\ Cubic Innovation Centre, Farringdon, London, UK
}

\begin{abstract}
Congestion at ticket gatelines is a growing problem. According to travel projections in the UK, the number of journeys for rail passengers is likely to double over the next 30 years. This creates the need to improve passenger throughput while still maintaining revenue protection. The proposed solution is to create a 'Gateless Gateline': a concept in which a user can seamlessly authenticate the intent to travel in train stations using current ticketing media ranging from barcode and Bluetooth low energy to radiofrequency identification, as well as new and emerging ticketing media, specifically related to biometrics. This article focuses on utilizing three-dimensional (3D) face recognition based on photometric stereo using near-infrared (NIR) light to illuminate a face for a 3D construction and tracking for the purpose of associating the correct biometric identifier with the correct person in a high-throughput environment. Other biometric systems have also been investigated as part of this project, i.e. palm vein scanning based on passing NIR light through the palm. There are challenges when it comes to associating passengers with valid travel tokens in a system which is free flowing and does not have a modus operandi of a standard access gate where validation occurs one at a time, normally signalled by paddles opening and closing. The proposed solution to the above, alongside the known issues associated with scaling biometric solutions, is reported in this article.
\end{abstract}

Keywords: biometrics, BLE, contactless, face recognition, Gateless Gateline, object tracking, machine learning, palm vein scanning, RFID

\section{INTRODUCTION}

Public transport ticketing may seem like a solved issue, but it is more important than most of us realize. While trains and tracks have looked mostly the same, ticketing has evolved throughout history to match the pace of our technological advancements. It has taken many forms: tokens, pieces of paper, magnetic strip cards, smart cards, until, finally, tickets are becoming embedded in credit cards and cell phones. This has already been implemented in London by TfL, where users can directly pay for their fares at the gates or on buses using contactless payment cards (CPCs) or cell phones embedded with near-field communication technology using Android Pay and Apple Pay. In the UK, over 81 million of such credit cards have been issued and their use in the public transport system has been increasing steadily, thanks also to some of the benefits for the users, such as special price caps, and the unification of ticketing, as CPCs can now be used on buses, tube, trains, bike shares and taxis [1]. The evolution of transport ticketing is migrating towards a unified and central account where various travel tokens are all linked. At the same time, as technology is developing in the field of biometric identification and its use to authenticate payments; it offers a prime opportunity to leverage biometric usage in the transportation industry and further evolve the transport ticketing arena. There are challenges with utilizing biometric technologies in a high-throughput and unpredictable (from a usability perspective) environment, which are discussed in this article.

The benefits associated with utilizing biometric technologies in the transportation industry include the opportunity to travel without any external ticket media and simply using a biometric marker such as your face, as a ticket token. 


\subsection{Gateless Gatelines}

Cubic's 'Gateless Gateline' is a concept for a new state-of-the-art gate for, but not limited to, use in mass transit systems. The premise is that it allows an unimpeded 'free flow' of passengers through the system, supporting a number of existing and new ticket media.

The concept of a free-flow fare gate works by first removing the point of validation (ticket inspection) from the gate, that is for it to be placed somewhere else on a station concourse. In addition, it offers the ability to increase the number of validation points as opposed to one 'validator per gate' which is the current state of the art for most fare gates. The removal of the validation from the fare gate ensures it becomes an unimpeded walkway where a passenger has no reason to stop or physically present a ticket to a validator.

The gate in this instance is used to validate passengers that it is expecting to 'see', using Bluetooth low energy (BLE), biometric detection and tracking which are explained in this report.

This project was partly funded under the 'Future Ticket Detection Competition' managed by the Rail Safety and Standards Board (RSSB). The scope of the competition was to 'identify future ticket detection technologies that could be used to accommodate growing passenger numbers and address infrastructure constraints in railway stations' with the aim 'to identify and demonstrate innovative solutions which tackle the following challenge themes:

- Passenger and ticket detection

- Checking for eligibility for travel

- Taking action for those with or without a valid ticket

- Throughput of passengers is also a significant area of challenge'

The system described in this report looks to address all the challenge themes and provide solution options for each.

\section{BIOMETRIC TRAVEL TOKENS}

The use of biometric identification in the public domain has increased as the technology, privacy and user acceptance have improved. The use of biometric technology on personal devices such as the iPhone has also helped the public to engage and trust the technology.

\subsection{Facial recognition}

The Gateless Gateline system relies on a key component which is its facial recognition capabilities. It is vital for revenue protection to ensure the facial recognition works in the conditions we are subjecting it to. Currently, paddles opening and closing based on a 1:1 ticket validation at the fare gate is the main revenue protection method. Design iterations to complement both the facial recognition requirements as well as the throughput and revenue protection requirements were fulfilled in this project.

The system has enabled facial recognition ticketing, i.e. the process of validating a registered face as a virtual ticket. Although there are a number of existing facial recognition approaches that can yield high recognition rates on publicly available databases (captured in controlled environments), their performance tends to degrade when applied to realistic environments. This is mainly due to the two-dimensional (2D) facial features utilized, which are volatile and subject to illumination and head pose variations. Consequently, 2D methods are deemed inadequate for scenarios such as crowded train stations with varying light conditions. 
A recent trend has been to incorporate three-dimensional (3D) information to aid recognition to combat the aforementioned challenges.

\subsubsection{Introduction to a photometric stereo-based facial recognition system}

To facilitate accurate and robust facial recognition in the Gateless Gateline system, a twosource photometric stereo (PS) system was proposed. A PS system employs a single camera and multiple illumination sources to capture greyscale images of an object illuminated from different angles. The greyscale images can then be used to reconstruct surface normals as a type of 3D feature. A representative PS-based facial recognition system - the photoface system previously developed [2] - employed four illumination sources and realized high recognition rates. However, the system proposed here only employs two light sources for reconstruction of (horizontal) surface gradients such that the practicability of the system is sufficient to suit Gateless Gateline scenarios. Near-infrared (NIR) lights are to be employed to make the system more covert and less intrusive. The surface gradient (3D) features have the advantages that they are independent of illumination and are robust to head poses to a large extent, meaning that they are most appropriate for accommodating dynamic passenger behaviours. Furthermore, being able to capture PS images and to reconstruct 3D features at a high frame rate makes this system superior to stereo vision systems in fulfilling the task of increasing the throughput in the aforementioned scenarios.

A 3D facial recognition system also has the advantage that it does not get 'spoofed' by a 2D photograph which cannot be discriminated from a real face by $2 \mathrm{D}$ feature-based systems.

\subsubsection{Construction of face databases}

Two types of face databases need to be constructed in order for registered users/passengers to be differentiated against those who are unregistered. A global face database will be constructed by passengers going to any station where an enrolment booth is available. Each passenger needs to be enrolled once (potentially periodically) in order to use the Gateless Gatelines. The hardware structure of the enrolment system will be similar to that of the facial recognition system, subject to minor adjustment (e.g. smaller in scale). This database is updated when a passenger gets registered or deregistered. Each local (per-station) face database is also constructed as a subset of the global face database. A local face database is updated when a passenger arrives at a station and is picked up (i.e. added to the database) via a BLE beacon which is linked to an application on a mobile device (see Section 4.1) or a passenger is successfully recognized by the facial recognition system while departing from a station (i.e. removed from the database).

\subsubsection{Requirements in a Gateless Gateline scenario}

A number of conditions are listed as follows such that the design of the facial recognition system can be more specific to the Gateless Gateline scenarios for a maximized performance.

- No strong backlight, i.e. direct lighting.

- Co-operative passengers, i.e. the passenger not intentionally avoiding the camera (i.e. no extreme head pose). Note that a passenger will not be required to stop and look up to the camera.

- No severe occlusion of the face to be recognized.

- A single-file channel for each gate.

- Persons will walk steadily through system (fast but not running/jogging). 
2.1.4 Facial recognition algorithm chain

The facial recognition system realizes its functionalities by following five main stages:

(a) Data capture: the system synchronizes a camera (referred to as camera $\mathrm{A}$ in the rest of the report) with two NIR light sources and captures two greyscale images of a gate corridor scene that is illuminated by the NIR lights, respectively.

(b) Face detection: it then detects for face regions in the greyscale images. An optional greyscale camera can be installed near camera A to assist the face detection process.

(c) Surface gradient recovery: for every face image present in a set of (two) PS images, the system performs a two-source PS algorithm that can recover (horizontal) surface gradient features.

(d) Feature processing: gradient features are then processed in order to have enhanced discriminative power.

(e) Facial recognition: discriminative features are compared to those in the local face database, and a face is classified as belonging to a particular passenger (i.e. a ticket holder) or otherwise an unenrolled/unregistered passenger (i.e. a potential fare evader).

Concerning the captured image frames, it should be noted that the facial recognition algorithm performs per-frame analysis rather than inter-frame tracking. This is a more robust option and can avoid the 'drifting' problem [3] that commonly exists in tracking methods, i.e. face tracking errors propagating to subsequent frames and causing failures beyond recovery. However, a limitation of per-frame analysis is that a previously recognized face cannot serve as prior knowledge for a subsequent frame, meaning that even if a face is recognized as belonging to a ticket holder in a certain image frame, it may be regarded as belonging to someone else in a subsequent frame. It should be noted that this has no impact on validating a virtual ticket (i.e. a registered face) and providing a confirmation to a passenger, but it poses a non-trivial problem to constructing a 'blacklist' for revenue protection. To resolve this issue, utilization of an overhead camera has been proposed to track passengers in a Gateless Gateline system. This camera can also be used to trigger the facial recognition camera by mapping coordinates of where the face was captured in relation to the place the person (object) is within the gateline.

\section{OBJECT TRACKING}

A key component of Gateless Gatelines is its ability to track users and to determine the status and validity of the tracked user.

\subsection{Validator (object) tracking}

Through in-depth user studies we understood how important feedback was to users when it comes to payment authentication. Users want to know whether payment has been successful or unsuccessful. There are certain challenges in a high-throughput transit environment when relying on wireless communications to achieve this repeatedly and accurately and that is why the system is designed with the object tracking application being the final 'decision-maker' and the biometric travel tokens (and any travel token) can feed into the system as individual modules.

\subsubsection{System functionality}

This section describes how the system works from the moment a user enters the station: (assuming they are enrolled with their biometric data and have an available payments account). 
- A user that has opted in for facial recognition receives feedback based on proximity beacons communicating with their app, where they will also receive visual and acoustic feedback at the gate.

- A non-account user using contactless ticketing media or a user with a registered palm who visits a validator would be 'tracked' as an object and followed to the gate and given visual and acoustic feedback accordingly. We are researching the potential to track users as they enter a 'validation zone'. At this point they will be tracked as an object. Once they have validated, the object, based on the Boolean output of the validator, will tag the object as successful or unsuccessful and this tag is aimed to be carried to the gate. As an object (person) enters a dedicated 'zone', they will receive a tracking ID. That tracking ID will remain with the object until they receive an output from the validator, where a 0 or 1 is added to the end of their tracking ID (based on successful, unsuccessful validation, respectively). The feedback loop is achieved when the person has walked through the Gateless Gateline. There is also logic on the validator which gives feedback if invalid facial data has been captured. This does not necessarily mean it will be invoked on the passenger as this could produce a negative mind-set towards the system (i.e. slower to validate than current systems), but more a way of continuously improving the design and usability of the validators. As long as a user has validated and can prove it; revenue operations do not get sacrificed. That is the extent to what a user does in the current system and any new system which is pro-posed should not require the user to have to do any more than they do currently.

\subsubsection{Account-based ticketing}

Current state, in its most simplified form, is a 'touch in, touch out' system. There are Bluetooth systems such as Check-In Be-Out that have also simplified the travelling experience for the end-user but as mentioned before, it requires some level of diligence from the user to ensure they have confirmed the start/end of their journey.

Our proposed solution for an account-based user is such that they can simply walk through a walkway-type gateline, without stopping and be charged correctly. The user would not be required to 'tap' or wait for paddles to open. The system being centred on tracking and facial recognition; the user simply has to get from validator to point of interest and their journey will be calculated accordingly (Fig. 1).

\subsubsection{Validator tracking software}

The software for the object tracking validation is standalone and employs multiple cameras for tracking. For the application to be useful for Gateless Gatelines, it has to be integrated with the overall system. This ensures that the system can produce a useful action, visible to the passenger, based on the output of the tracking software.

The software modules will communicate with each other using HTTP-based requests. An example application of a HTTP request will be to get user validation output from the tracking software and for the other software module to trigger an LED to turn on and off based on the output it got from the tracking software, i.e. tracking software produces X result, gate software sees the $\mathrm{X}$ result and triggers another programme to execute.

As shown in Fig. 2 the tracking software application will post data to a server and the gate application will retrieve that data in a format that it can interpret and utilize to interact with the passenger. 


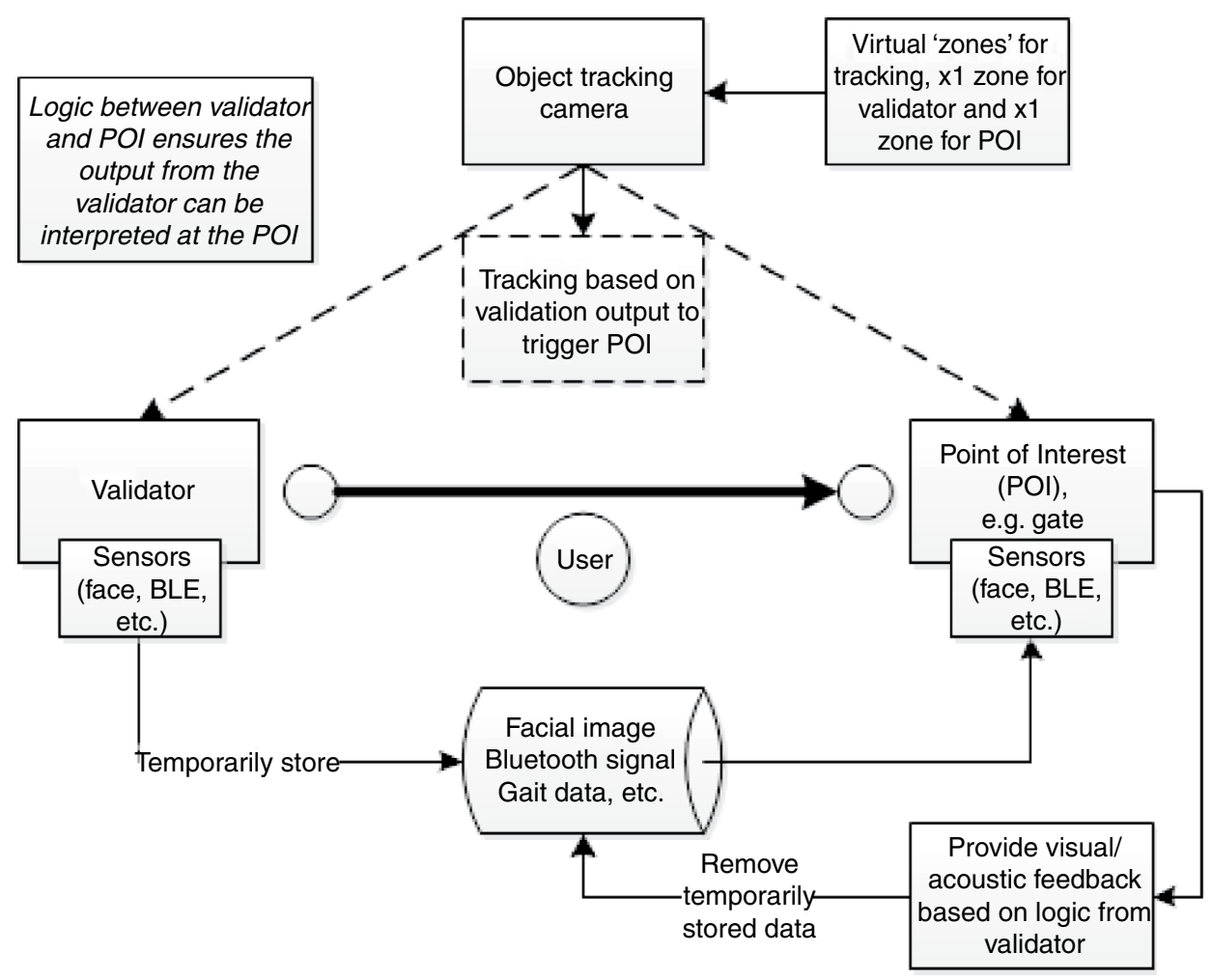

Figure 1: Validator-gate-tracking schematic.

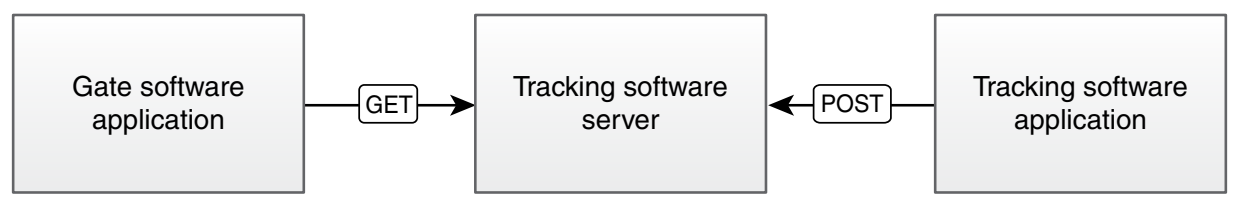

Figure 2: Structure of HTTP requests for tracking application.

\subsubsection{Tracking software interfaces}

The facial recognition software will store recognized biometric data and consequently its corresponding account that it belongs to.

- The facial recognition software records coordinates (face relative to camera).

- The distance from face to the camera will also be recorded

- A RESTful API obtains these parameters, thus feeding the data to the object tracking application as shown in Fig. 2.

- Once the coordinates of a valid image is retrieved; the object tracker can produce a valid output on that particular object.

There needs to be a level of logic and calculation as to what coordinates to pull as a moving passenger's coordinate will constantly change. Adding an amount (to be 
continuously optimized) to $X$ and $Y$, based on average walking speed of person vs. time it takes for object tracking application to pick up the coordinates recorded in the file will improve the tracking capabilities in crowded areas.

- The object tracking application makes the final decision on every user (object) passing through the detection zone (gate end-point). Whether the system pulls that information from the facial recognition system or output received from a validator.

- All the interfaces are REST ful API's.

- All data exchanges between subsystems will be based on JSON schema, so can be easily extended and parsed.

- All server elements will be based on node.js, allowing for a quick development cycle, utilization of components developed in the open-source community and great portability.

\subsection{Tracking conclusion}

Initially the system was designed with facial recognition being at the core of the system architecture and the object tracking system was there to aid other users during validation. The consequent success of this approach led to an idea of utilizing tracking at the core of the system with any payment token being a module that plugs into the tracking application.

The tracking application captures every person (object) going through and tags them as a neutral object (unrecognized) - from a technical aspect, it does nothing more than this but the intelligence comes from feeding useful data sources to the object tracking system. The validator application and the facial recognition system will prompt the object tracking system to tag specific objects depending on what the consequent systems have captured, i.e. the facial recognition system picks up a registered individual and sends their coordinates to the object tracking camera, this system then tags that object as a 'valid object' until it has passed through a point of interest (in this case the gateline). Similar principles apply with the validator; a user who successfully validates at a validator gets directly tagged by the object tracking camera (with the assumption that the object tracking camera has a field of view that contains both the validator and the gate. There will be further investigations to realize the feasibility of combining cameras for a greater field of view and what this does to the accuracy of the object tracking application).

\section{SCALABLE BIOMETRIC SOLUTIONS}

The limitation of biometric data in high-throughput environments is the size of the database and the consequent speed and accuracy of successfully identifying a match. There are database solutions that can store millions of travel tokens, but to scan a database of e.g. 10,000 users would take longer than it takes to validate a travel token using contactless radiofrequency identification (RFID) technology. The Gateless Gateline system has to work for millions of users. The scanning, recognition and identification has to happen at fractions of a second to realistically compete with current travel tokens such as barcode and RFID technologies.

\subsection{Dynamic databases using BLE}

One of the technical challenges in this project is where data has to be retrieved in real time while a passenger is approaching the gate. If every passenger that opted in for the Gateless Gateline service had their account/biometric information stored on a central database, the 
system would take too long to down-select the person, also, the larger the database, the more likely the chance of incorrectly matching biometric data.

To overcome this problem, the system will look to use BLE to connect to the passengers within close proximity to the gate and down-select the database when the passengers are ' $\mathrm{X}$ ' metres away from the actual gate. This allows the system to know who is about to approach the gate (in any order) as the gate will communicate with the passengers' BLE-enabled app on their mobile device.

To enable this functionality, passengers would need to store their travel tokens onto their BLE-enabled mobile devices to allow the gate to communicate with their devices prior to entering the gate, allowing the system to only try and match one passenger against the few passengers around them and the gate, not only making it more accurate but also a lot quicker than to scan a database of millions of passengers.

\subsection{Dynamic databases using machine learning}

The various technologies (below) are used to perform a database pre-population process. The systems shown all require an account-based solution except the machine learning (ML) system which can use both. The premise is to use various forms of ML to pre-populate a realtime and dynamic database. This means the user is not required to perform an initial task or rely on a process to have occurred such as BLE communicating with their mobile devices (as mentioned in Section 4.1) and populating the database through an intermediary process. All the methods below are novel ways of pre-populating a real-time database but for this particular solution, we will be focusing on the ML aspect which only relies on the user to be present at a train station and for the model to predict a passenger's travel pattern.

The ML capability to pre-populate a database could expand to prediction of group travellers, i.e. one passenger is detected and the rest of the 'usual group' surrounding that passenger also gets pre-populated.

The process for ML for real-time predictive analytics is as follows:

- Setting up a source (the data which will be collected from the gate - UUID, date, time, image, account information (if opted-in for), WiFi MAC address).

- Creating a dataset from the data collected above.

- Creating a model by turning the dataset into an actionable and visual predictive model.

- Generating predictions by inputting new data to get immediate predictions based on the created model (Fig. 3).

For the predictive model to work as shown in Fig. 3, the user would initially have to follow step (1) and present their token to a validator (where an image (potentially) and time stamp of the passenger is also captured) numerous times to create a large dataset to make accurate predictions. Once that is complete, the user would follow step (2). There is scope for more innovation around how this information is communicated to the passenger: the time limit before the local database removes the user and how this is communicated with the passenger - countdown timer; etc.

How the theory would work with a biometric travel token which is registered for a monthly ticket: register the user after ' $x$ ' amount of times and they only have to show the validity of their ticket once a month, i.e. to ensure that a monthly user has presented a valid monthly ticket instead of becoming 'trusted' after one month and then never having to prove a valid ticket again. Instead the user would, for example, show a ticket for a whole month, become 


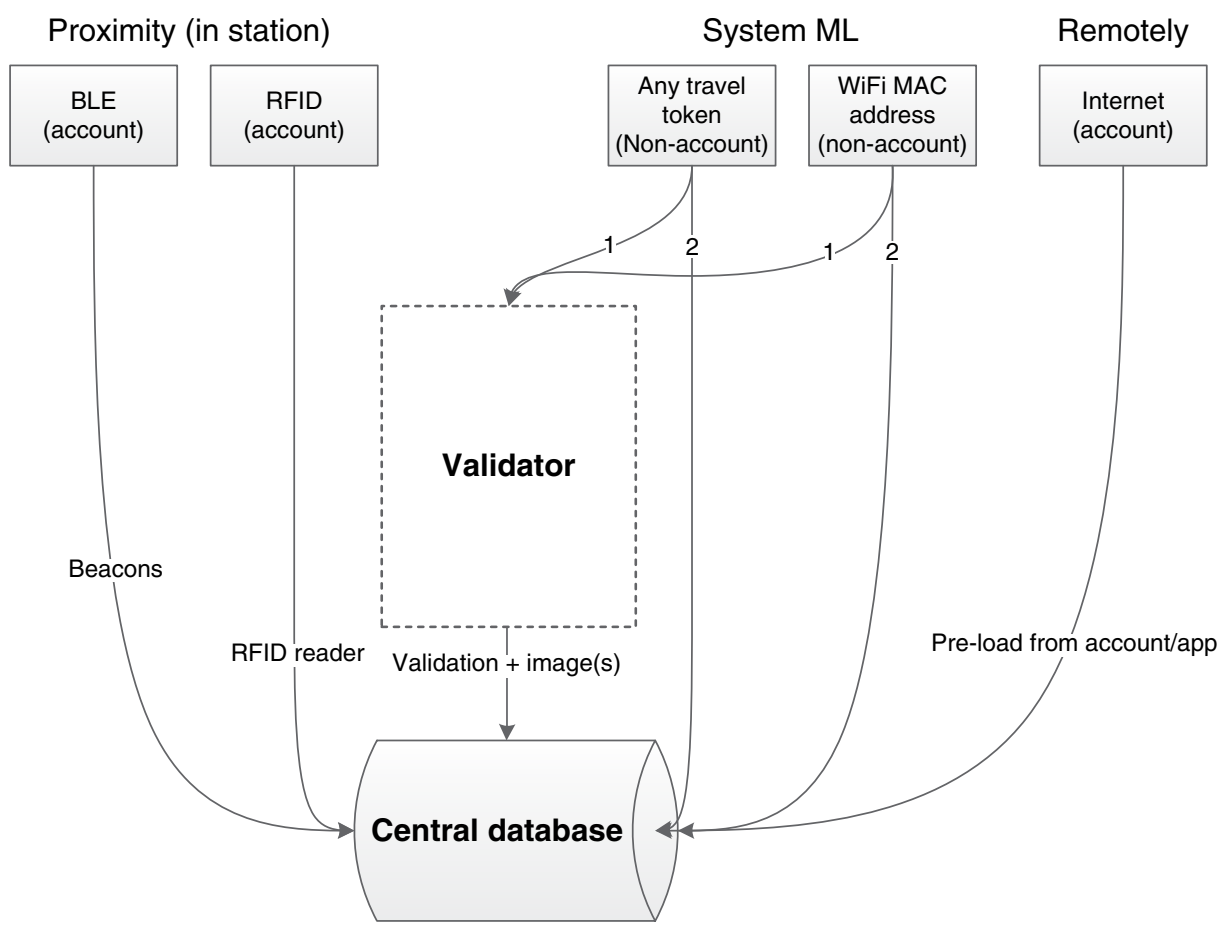

Figure 3: Machine learning for Gateless Gatelines.

trusted, then only show a ticket once a month for the remaining 11 months of the year to prove they have a monthly ticket.

An account-based user could be notified through their app, email, etc. that they have been 'seen' by the system enough to create a good predictive model based on their historic travel patterns.

The same principles apply; based on the type of ticket (monthly or yearly), the user will have to present a valid ticket at some point in that cycle to re-trigger the validation process. Investigation will need to be made as to whether this would be on a set date or random. The account-based user would bypass this as they can be automatically debited through the automated fare collection system which is uniquely matched to their biometric data.

\section{CONCLUSION}

This article has explored a variety of technologies that has the potential to be used in a system such as a Gateless Gateline concept. The encouraging factor from early trials is the ability to differentiate the false-positive results when comparing 3D and 2D facial recognition, whereas 2D recognition can be fooled by printed imaging, 3D recognition cannot.

The ability to recognize individuals while walking and not directly looking at the facial recognition camera was also a promising result. In fact, the system achieved to capture an individual walking at $\sim 6.5 \mathrm{~km} / \mathrm{h}$ while their head was tilted $\sim 45$ degrees downwards looking at their mobile device. There are studies to be conducted where the enrolment process (where a number of different head poses are captured for a specific person to create their biometric 
profile) is optimized based on user behavioural studies, i.e. understanding what the most common head poses are when going through a Gateless Gateline and to what extent this could be influenced by design of the system.

In a system where individual feedback is required to passengers and for operators to understand when the system is being misused, tracking was proven to be an integral component overlooking the whole system. By utilizing tracking, the system knows the validity status of every person walking through the gate, whether they are registered or not, the system will validate accordingly, thus removing the requirement to perform a 1:1 ticket inspection at every fare gate.

As explained in Section 4.1; BLE is used as an intermediary to pre-populate a database of registered users who are within close proximity to the Gateless Gateline. There is testing to be performed based on BLE in crowded environments and what happens to the signal strength when a mobile device is covered with clothing, items, etc.

Deploying biometric solutions at scale has been and continues to be, a global barrier. The requirement is therefore a two-form validation process such as facial recognition alongside a passport scan currently deployed in many airports.

In a railway environment, there is the opportunity to deploy a fully biometric system with no secondary identifier. The reason for this being, as mentioned in Section 4.2, that rail passengers (specifically commuters) are more likely to follow a regular travel pattern in terms of time, origin and destination which feeds well into an ML algorithm that can predict the time and date that passengers will be travelling and pre-populating a database based on historic information as opposed to an external identifier such as a mobile device, ultimately transforming the Gateless Gateline into a full biometric system.

\section{ACKNOWLEDGEMENTS}

This work was partly funded by the RSSB under the 'Future Railway' call on behalf of the Technical Strategy Leadership Group.

The partners in this project were Bristol Robotics Laboratory, Maynard Designs, Movement Strategies and Go Ahead group.

\section{REFERENCES}

[1] Neves, C., The Evolution of Public Transport Ticketing, Iglus.org. N.p., 2017.

[2] Hansen, M.F., Atkinson, G.A., Smith, L.N. \& Smith, M.L., 3D face reconstructions from photometric stereo using near infrared and visible light. Computer Vision and Image Understanding, 114(8), pp. 942-951, 2010. DOI: 10.1016/j.cviu.2010.03.001.

[3] Grabner, H., Grabner, M. \& Bischof, H., Real-time tracking via on-line boosting. British Machine Vision Conference, 1(5), p. 6, 2006. DOI: 10.5244/C.20.6. 